\title{
Noncyclotomic $\mathbb{Z}_{p}$-Extensions of Imaginary Quadratic Fields
}

\author{
Takashi Fukuda and Keiichi Komatsu
}

\section{CONTENTS}

\section{Introduction}

2. Criteria

3. Construction of $K_{n}$

4. Computation of $K_{2}$

5. Experimentation for $p=3$

References

2000 AMS Subject Classification: Primary 11G15, 11R27, 1140

Keywords: Iwasawa invariants, Siegel function, computation
Let $p$ be an odd prime number which splits into two distinct primes in an imaginary quadratic field $K$. Then $K$ has certain kinds of noncyclotomic $\mathbb{Z}_{p}$-extensions which are constructed through ray class fields with respect to a prime ideal lying above $p$. We try to show that Iwasawa invariants $\mu$ and $\lambda$ both vanish for these specfic noncyclotomic $\mathbb{Z}_{p}$-extensions.

\section{INTRODUCTION}

Let $p$ be a prime number. Then the rational number field $\mathbb{Q}$ has the unique $\mathbb{Z}_{p}$-extension $\mathbb{Q}_{\infty}$. Iwasawa proved elegantly that the class numbers of all intermediate fields of $\mathbb{Q}_{\infty} / \mathbb{Q}$ are prime to $p$ ([Iwasawa 56$]$ ). Consequently, Iwasawa invariants $\mu\left(\mathbb{Q}_{\infty} / \mathbb{Q}\right)$ and $\lambda\left(\mathbb{Q}_{\infty} / \mathbb{Q}\right)$ are both zero. This is based on the fact that there is a unique prime ideal of $\mathbb{Q}$ ramified in $\mathbb{Q}_{\infty}$ which is totally ramified. Our purpose in this paper is to consider a noncyclotomic ana$\log$ to Iwasawa's theorem in the case where the base field is an imaginary quadratic field. We give some numerical evidence for our expectation.

Let $K$ be an imaginary quadratic field and $p$ an odd prime number which splits into two distinct primes $\mathfrak{p}$ and $\overline{\mathfrak{p}}$ in $K$. We denote by $K_{n}^{\prime}=K\left(\mathfrak{p}^{n+1}\right)$ the ray class field of $K$ modulo $\mathfrak{p}^{n+1}$ and put $K_{\infty}^{\prime}=\cup_{n=0}^{\infty} K_{n}^{\prime}$. Then there exists a unique $\mathbb{Z}_{p}$-extension $K_{\infty}$ of $K$ in $K_{\infty}^{\prime}$. In the same way as $\mathbb{Q}_{\infty} / \mathbb{Q}$, there is a unique prime ideal of $K$ which is ramified in $K_{\infty}$. One of the differences is that the prime $\mathfrak{p}$ of $K$ is not always totally ramified in $K_{\infty}$. We are led to the following problem.

Problem 1.1. If $\mathfrak{p}$ is totally ramified in $K_{\infty}$ over $K$, do the Iwasawa invariants $\mu\left(K_{\infty} / K\right)$ and $\lambda\left(K_{\infty} / K\right)$ vanish?

We note that our situation can be also considered as an analog to Greenberg's conjecture which states that both $\mu$ and $\lambda$ vanish for the cyclotomic $\mathbb{Z}_{p}$-extension of any totally real number field. Since an imaginary quadratic

(c) A K Peters, Ltd 1058-6458/2001 $\$ 0.50$ per page Experimental Mathematics 11:4, page 469 
field has no nontrivial units, our situation is simpler even in comparison with Greenberg's conjecture for the real quadratic case. We hope that studies of this problem provide a somewhat new approach to the original conjecture of Greenberg.

\section{CRITERIA}

We begin with some notation. Let $k$ be an algebraic number field. We denote by $\mathfrak{O}_{k}$ the integer ring of $k$, by $I_{k}$ the ideal group of $k$, by $P_{k}$ the principal ideal subgroup of $I_{k}$, and by $h_{k}$ the class number of $k$. Let $L$ be a Galois extension of $k$. We denote by $G(L / k)$ the Galois group of $L$ over $k$ and $N_{L / k}$ the norm mapping of $L$ over $k$.

Now, as mentioned before, let $K$ be an imaginary quadratic field and $p$ an odd prime number which splits into two distinct primes $\mathfrak{p}$ and $\overline{\mathfrak{p}}$ in $k$. We denote by $K_{n}^{\prime}=K\left(\mathfrak{p}^{n+1}\right)$ the ray class field of $K$ modulo $\mathfrak{p}^{n+1}$ and put $K_{\infty}^{\prime}=\cup_{n=0}^{\infty} K_{n}^{\prime}$. Then there exists a unique $\mathbb{Z}_{p}$-extension $K_{\infty}$ of $K$ in $K_{\infty}^{\prime}$. We set $\Gamma=G\left(K_{\infty} / K\right)$.

Let $K_{n}$ be the $n$-th layer of $K_{\infty}$ over $K, A_{n}$ the $p$ primary part of the ideal class group of $K_{n}, B_{n}=A_{n}^{\Gamma}=$ $\left\{c \in A_{n} \mid c^{\sigma}=c\right.$ for any $\left.\sigma \in \Gamma\right\}, B_{n}^{\prime}$ the subgroup of $A_{n}$ consisting of ideal classes containing ideals invariant under the action of $G\left(K_{n} / K\right)$, and $D_{n}$ the subgroup of $A_{n}$ consisting of classes which contain an ideal, all of whose prime factors lie above $\mathfrak{p}$. Note that the definition of $D_{n}$ here is different from that in [Greenberg 76]. If $m \geq n$, we can define a homomorphism $i_{n, m}: A_{n} \rightarrow A_{m}$ by sending the ideal class $\operatorname{cl}(\mathfrak{a})$ to $\operatorname{cl}\left(\mathfrak{a} \mathfrak{O}_{K_{m}}\right)$ for any ideal $\mathfrak{a}$ of $K_{n}$. We set $H_{n, m}=\operatorname{Ker} i_{n, m}$. We also define a homomorphism $N_{m, n}: A_{m} \rightarrow A_{n}$ by sending the ideal class $\operatorname{cl}(\mathfrak{a})$ to $\operatorname{cl}\left(N_{k_{m} / k_{n}}(\mathfrak{a})\right)$ for any ideal $\mathfrak{a}$ of $K_{m}$. Moreover, we denote by $\lambda_{p}$ and $\mu_{p}$ the Iwasawa invariants of the $\mathbb{Z}_{p}$-extension $K_{\infty} / K$. It is well known that $\mu_{p}=0$ by [Gillard 85] and [Schneps 1987]. On the other hand, few results are known about $\lambda_{p}$.

We concentrate our attention on the case where $\mathfrak{p}$ is totally ramified in $K_{\infty}$. If $h_{K}$ is prime to $p$, then $\lambda_{p}=0$ by Iwasawa's theorem [Iwasawa 56]. So we are interested in the case $A_{0} \neq 0$. We first note that the order of $B_{n}$ is explicitly known because $K$ has no nontrivial units. The following lemma is the direct consequence of the genus formula ([Yokoi 1967]).

Lemma 2.1. Assume that $\mathfrak{p}$ is totally ramified in $K_{\infty}$ over $K$. Then, $\left|B_{n}\right|=\left|A_{0}\right|$ for all $n \geq 0$.

The following proposition is the fundamental criterion for $\lambda_{p}=0$. Though the proof is essentially the same as in [Greenberg 76, Theorem 2], we include a proof as a convenience.

Proposition 2.2. Assume that $\mathfrak{p}$ is totally ramified in $K_{\infty}$ over $K$. Then $\mu_{p}=\lambda_{p}=0$ if and only if $B_{n}=D_{n}$ for some integer $n \geq 0$.

Proof: Assume $B_{n}=D_{n}$ and let $m \geq n$. Since the prime of $k_{n}$ lying over $\mathfrak{p}$ is totally ramified in $k_{m}$, both $N_{m, n}$ : $A_{m} \rightarrow A_{n}$ and $N_{m, n}: D_{m} \rightarrow D_{n}$ are surjective. Then Lemma 2.1 implies the injectivity of $N_{m, n}: B_{m} \rightarrow B_{n}$ and hence, the injectivity of $N_{m, n}: A_{m} \rightarrow A_{n}$, which means $\left|A_{m}\right|=\left|A_{n}\right|$. Hence, $\mu_{p}=\lambda_{p}=0$. Conversely, assume $\mu_{p}=\lambda_{p}=0$. Then $A_{0}=H_{0, n}$ for some $n \geq 0$ ([Greenberg 76, Proposition 2]). Hence, the genus formula yields $B_{n}=B_{n}^{\prime}=i_{0, n}\left(A_{0}\right) D_{n}=D_{n}$.

Corollary 2.3. Assume that $\mathfrak{p}$ is totally ramified in $K_{\infty}$ over $K$. Then $\mu_{p}=\lambda_{p}=0$ if and only if every ideal class of $A_{0}$ becomes principal for some $n \geq 0$. [Minardi 86]

Proof: Assume $A_{0}=H_{0, n}$ for some $n \geq 0$. Then the genus formula yields $B_{n}=B_{n}^{\prime}=i_{0, n}\left(A_{0}\right) D_{n}=D_{n}$. Hence, $\mu_{p}=\lambda_{p}=0$ by Proposition 2.2. The converse is a part of [Greenberg 76, Proposition 2].

As an application of Proposition 2.2, we have the following proposition. We note that for Proposition 2.4, $\mu_{p}=\lambda_{p}=0$ even when $\mathfrak{p}$ is not totally ramified in $K_{\infty}$.

Proposition 2.4. If $h_{K}=p$, then $\mu_{p}=\lambda_{p}=0$.

Proof: If the initial layer $K_{1}$ of $K_{\infty}$ over $K$ is the absolute class field of $K$, then $\lambda_{p}=0$ by the genus formula. Assume that $\mathfrak{p}$ is totally ramified in $K_{\infty}$. Since $h_{K}=p$, there exists a prime number $q$ with $q \equiv 3(\bmod 4)$ such that $K=\mathbb{Q}(\sqrt{-q})$. Let $\chi$ be a Dirichlet character associated to $K$. Then, since $\left(\frac{-1}{q}\right)=-1$, we have

$$
\begin{aligned}
p & =h_{K}=\frac{1}{q} \sum_{\nu=1}^{q-1} \chi(\nu) \nu=\frac{1}{q} \sum_{\nu=1}^{\frac{q-1}{2}}(\chi(\nu) \nu-\chi(\nu)(q-\nu)) \\
& =\frac{1}{q} \sum_{\nu=1}^{\frac{q-1}{2}} \chi(\nu)(2 \nu-q) \leq \frac{1}{q} \sum_{\nu=1}^{\frac{q-1}{2}}(q-2 \nu)=\frac{(q-1)^{2}}{4 q}<\frac{q}{4} .
\end{aligned}
$$

We assume that $\mathfrak{p}$ is a principal ideal of $K$. Then there exist integers $x, y \in \mathbb{Z}$ with $\mathfrak{p}=\left(\frac{x+y \sqrt{-q}}{2}\right)$, which implies that $p=\frac{x^{2}+y^{2} q}{4}<\frac{q}{4}$. This is a contradiction. Hence, we have $D_{0}=A_{0}$, and thus $\mu_{p}=\lambda_{p}=0$ by Proposition 2.2 . 
In Sections 4 and 5, we apply Proposition 2.2 and Corollary 2.3 for $K_{n}$ constructed explicitly by computer when $p=3$. For that, the discriminant $d\left(K_{n}\right)$ of $K_{n}$ is needed.

Lemma 2.5. $d\left(K_{n}\right)=p^{\left(p^{n}-1\right)\left(n+1-\frac{1}{p-1}\right)+n} d\left(K_{0}\right)^{p^{n}}$.

Proof: Apply the conductor-discriminant formula for $K_{n} / K_{0}$.

\section{CONSTRUCTION OF $K_{n}$}

We use the same notation as in Section 2. We explain a method for constructing $K_{n}$ using complex multiplication for an odd prime number $p$ and an imaginary quadratic field $K$ different from $\mathbb{Q}(\sqrt{-1})$ and $\mathbb{Q}(\sqrt{-3})$. It is well known that an abelian extension of an imaginary quadratic field is generated by a special value of the $j$-function, but the $j$-function produces polynomials with huge coefficients and is not useful in actual computations. There are several methods to find polynomials which generate a ray class field of an imaginary quadratic field and have small coefficients using Weber function or Weierstrass $\sigma$-function ([Schertz 97], [Stevenhagen 2001]). We shall provide a similar, but slightly different, approach using Siegel functions.

First we define Siegel functions: Let $a_{1}, a_{2}$ be rational numbers and $\tau$ a complex number with positive imaginary parts. The Siegel functions are defined by

$$
\begin{gathered}
g\left(a_{1}, a_{2}\right)(\tau)=-q_{\tau}^{(1 / 2)\left(a_{1}^{2}-a_{1}+1 / 6\right)} e^{2 \pi i a_{2}\left(a_{1}-1\right) / 2}\left(1-q_{z}\right) \\
\cdot \prod_{n=1}^{\infty}\left(1-q_{\tau}^{n} q_{z}\right)\left(1-q_{\tau}^{n} q_{z}^{-1}\right),
\end{gathered}
$$

where $q_{\tau}=e^{2 \pi i \tau}, q_{z}=e^{2 \pi i z}$ and $z=a_{1} \tau+a_{2}$. Then $g\left(a_{1}, a_{2}\right)(\tau)$ is a modular function of some level and $K_{n}$ is generated using special values of $g$.

Let $I_{\mathfrak{p}}$ be the subgroup of $I_{K}$ generated by the ideals which are prime to $\mathfrak{p}$. We put $S_{\mathfrak{p}^{n}}=\left\{(\alpha) \in P_{K} \mid \alpha \equiv 1\right.$ $\left.\left(\bmod \mathfrak{p}^{n}\right)\right\}$. Let $C$ be an element of the ray class group $I_{\mathfrak{p}} / S_{\mathfrak{p}^{n+1}}$. We call $C$ a ray class modulo $\mathfrak{p}^{n+1}$ in $K$. Let $\mathfrak{c}$ be an ideal of $C$ and denote $C$ by $\operatorname{cl}_{n+1}(\mathfrak{c})$. Then there exist elements $\omega_{1}, \omega_{2}$ in $K$ with $\operatorname{Im}\left(\omega_{1} / \omega_{2}\right)>0$ such that $\mathfrak{p}^{n+1} \mathfrak{c}^{-1}=\mathbb{Z} \omega_{1}+\mathbb{Z} \omega_{2}$. Since $(p)=\mathfrak{p} \overline{\mathfrak{p}}$, there exist integers $r, s \in \mathbb{Z}$ with $\frac{r}{p^{n+1}} \omega_{1}+\frac{s}{p^{n+1}} \omega_{2}=1$. We set

$$
g_{\mathfrak{p}^{n+1}}(C)=g\left(\frac{r}{p^{n+1}}, \frac{s}{p^{n+1}}\right)\left(\frac{\omega_{1}}{\omega_{2}}\right)^{12 p^{n+1}}
$$

which depends only on $C$ by [Kubert and Lang 81, page 33, Proposition 1.3]. Then $g_{\mathfrak{p}^{n+1}}(C)$ is in $K_{n}^{\prime}=$
$K\left(\mathfrak{p}^{n+1}\right)$ by [Kubert and Lang 81 , page 234 , Theorem 1.1] and $\left(g_{\mathfrak{p}^{n+1}}(C)\right)=\mathfrak{p}_{n}^{\prime 6 p^{n+1}}$ by [Kubert and Lang 81, page 246, Theorem 3.2], where $\mathfrak{p}_{n}^{\prime}$ is the prime ideal of $K_{n}^{\prime}$ lying over $\mathfrak{p}$. Let $S$ be a ray class modulo $\mathfrak{p}^{n+1}$ in $K$. Then we have

$$
g_{\mathfrak{p}^{n+1}}(C)\left(\frac{K_{n}^{\prime} / K}{S}\right)=g_{\mathfrak{p}^{n+1}}(S C)
$$

by [Kubert and Lang 81, page 234, Theorem 1.1], where $\left(\frac{K_{n}^{\prime} / K}{S}\right)$ is the Artin symbol of $S$. In particular, if we set $\sigma=\left(\frac{K_{n}^{\prime} / K}{1+p}\right)$, then

$$
g_{\mathfrak{p}^{n+1}}(C)^{\sigma}=g\left(\frac{r(1+p)}{p^{n+1}}, \frac{s(1+p)}{p^{n+1}}\right)\left(\frac{\omega_{1}}{\omega_{2}}\right)^{12 p^{n+1}} .
$$

We use the following lemmas for our computation.

Lemma 3.1. Let $\mathrm{cl}_{0}\left(\mathfrak{a}_{1}\right), c l_{0}\left(\mathfrak{a}_{2}\right), \cdots, \operatorname{cl}_{0}\left(\mathfrak{a}_{r}\right)$ be generators of $A_{0}, p^{e_{i}}>1$ the order of $\operatorname{cl}_{0}\left(\mathfrak{a}_{i}\right)$ and $\widetilde{K}$ the absolute class group of $K$. We suppose that there exists an element $\alpha_{i}$ in $\mathfrak{O}_{K}$ with $\mathfrak{a}_{i}^{p^{e_{i}}}=\left(\alpha_{i}\right)$, such that $\alpha_{i} \equiv 1$ $\left(\bmod \mathfrak{p}^{e_{i}+1}\right)$. Then $\widetilde{K} \cap K_{n}=K$ and there exist ideals $\mathfrak{a}_{1}^{\prime}, \mathfrak{a}_{2}^{\prime}, \cdots, \mathfrak{a}_{r}^{\prime}$ of $K$ with $\operatorname{cl}_{0}\left(\mathfrak{a}_{i}\right)=\operatorname{cl}_{0}\left(\mathfrak{a}_{i}^{\prime}\right)$, such that the orders of $\mathrm{cl}_{n+1}\left(\mathfrak{a}_{n}^{\prime}\right)$ are $p^{e_{i}}$, respectively.

Proof: Since $\alpha_{i} \equiv 1\left(\bmod \mathfrak{p}^{e_{i}+1}\right)$ and since $(1+p) S_{\mathfrak{p}^{n+1}}$ is a generator of $S_{\mathfrak{p}} / S_{\mathfrak{p}^{n+1}}$, there exists an integer $s \in \mathbb{Z}$ with $(1+p)^{p^{s e_{i}}} \alpha_{i} \equiv 1\left(\bmod \mathfrak{p}^{n+1}\right)$. We put $\mathfrak{a}_{i}^{\prime}=\mathfrak{a}_{i}(1+$ $p)^{s}$. Then $\operatorname{cl}_{0}\left(\mathfrak{a}_{i}\right)=\operatorname{cl}_{0}\left(\mathfrak{a}_{i}^{\prime}\right)$ and the order of $\operatorname{cl}_{n+1}\left(\mathfrak{a}_{i}^{\prime}\right)$ is $p^{e_{i}}$. If the order $m$ of $\operatorname{cl}_{1}(\mathfrak{a})$ is prime to $p$ for some ideal $\mathfrak{a}$, then there exists an integer $\alpha$ of $K$ such that the order of $\mathrm{cl}_{n+1}(\mathfrak{a}(\alpha))$ is $m$. This shows that $\widetilde{K} \cap K_{n}=K$.

Lemma 3.2. Let $C_{0}$ be the ray class of modulo $\mathfrak{p}^{n+1}$ with $C_{0}=\operatorname{cl}_{n+1}\left(\mathfrak{O}_{K}\right), \sigma=\left(\frac{K_{n}^{\prime} / K}{1+p}\right)$ the Artin symbol and set

$$
\alpha=N_{K_{n}^{\prime} / K_{n}}\left(g_{\mathfrak{p}^{n+1}}\left(C_{0}\right)^{1-\sigma}\right) .
$$

Then there exists a unique element $\beta$ of $K_{n}$ with $\beta^{3 p^{n+1}}=$ $\alpha$ such that $K_{n}=K(\beta)$. Furthermore, $\beta$ is a unit of $K_{n}$.

Proof: Let $\omega_{1}$ and $\omega_{2}$ be a basis of $\mathfrak{p}^{n+1}$ over $\mathbb{Z}$ with $\operatorname{Im}\left(\omega_{1} / \omega_{2}\right)>0$. Then there exist integers $r, s \in \mathbb{Z}$, such that $\frac{r}{p^{n+1}} \omega_{1}+\frac{s}{p^{n+1}} \omega_{2}=1$. Hence we have

$$
g_{\mathfrak{p}^{n+1}}\left(C_{0}\right)=g\left(\frac{r}{p^{n+1}}, \frac{s}{p^{n+1}}\right)\left(\frac{\omega_{1}}{\omega_{2}}\right)^{12 p^{n+1}}
$$

and

$$
\begin{aligned}
& g_{\mathfrak{p}^{n+1}}\left(\operatorname{cl}_{n+1}((1+p)) C_{0}\right)= \\
& g\left(\frac{r(1+p)}{p^{n+1}}, \frac{s(1+p)}{p^{n+1}}\right)\left(\frac{\omega_{1}}{\omega_{2}}\right)^{12 p^{n+1}} .
\end{aligned}
$$


Since the quotient

$f(\tau)=\left(g\left(\frac{r}{p^{n+1}}, \frac{s}{p^{n+1}}\right)(\tau) / g\left(\frac{r(1+p)}{p^{n+1}}, \frac{s(1+p)}{p^{n+1}}\right)(\tau)\right)^{4}$

of Siegel functions is a modular function of level $p^{2 n+2}$ whose $q$-expansion at $\infty$ has coefficients in $\mathbb{Z}\left[\zeta_{p^{2 n}}\right]$, $f\left(\omega_{1} / \omega_{2}\right)$ is in $K\left(p^{2 n+2}\right)$ by [Stark 1980, Theorem 3]. We assume $a=f\left(\omega_{1} / \omega_{2}\right)^{3 p^{m}} \in K\left(\mathfrak{p}^{n+1}\right)$ and $X^{p}-a$ is irreducible over $K\left(\mathfrak{p}^{n+1}\right)$. Since $K\left(\mathfrak{p}^{n+1}\right)\left(f\left(\omega_{1} / \omega_{2}\right)\right)$ is an abelian extension of $K$, we have $K\left(\mathfrak{p}^{n+1}\right) \varsubsetneqq$ $K\left(\mathfrak{p}^{n+1}\right)\left(\zeta_{p}\right) \subset K\left(\mathfrak{p}^{n+1}\right)\left(f\left(\omega_{1} / \omega_{2}\right)^{3 p^{m-1}}\right)$ since $\zeta_{p} \notin$ $K\left(\mathfrak{p}^{n+1}\right)$. This is a contradiction. Hence, we have $f\left(\omega_{1} / \omega_{2}\right)^{3} \zeta \in K\left(\mathfrak{p}^{n+1}\right)$ for some $p^{n+1}$-th root of unity $\zeta$. Moreover, we have $f\left(\omega_{1} / \omega_{2}\right) \zeta^{\prime} \in K\left(\mathfrak{p}^{n+1}\right)$ for some $3 p^{n+1}$-th root of unity $\zeta^{\prime}$ since $\zeta_{3} \notin K\left(\mathfrak{p}^{n+1}\right)$.

We now make some comments about the numerical calculation of Siegel functions. Let $\mathfrak{c}$ be an ideal of a ray class $C$. We choose a basis $\left\{\omega_{1}, \omega_{2}\right\}$ of $\mathfrak{p}^{n+1} \mathfrak{c}^{-1}$ so that $\omega_{1} / \omega_{2}$ belongs to the fundamental domain for $\mathrm{SL}_{2}(\mathbb{Z})$ for rapid convergence of $g\left(a_{1}, a_{2}\right)\left(\omega_{1} / \omega_{2}\right)$. It is also important to adjust $a_{i}$ so that $0 \leq a_{i}<1$ by

$$
\begin{aligned}
& g\left(a_{1}+n_{1}, a_{2}+n_{2}\right)(\tau)= \\
& \quad(-1)^{n_{1} n_{2}+n_{1}+n_{2}} e^{\pi i\left(n_{2} a_{1}-n_{1} a_{2}\right)} g\left(a_{1}, a_{2}\right)(\tau) \quad\left(n_{i} \in \mathbb{Z}\right) .
\end{aligned}
$$

\section{COMPUTATION OF $K_{2}$}

For $p=3$ and several $K \mathrm{~s}$, we constructed $K_{1}$ and $K_{2}$ explicitly by computer and examined whether $H_{0, n}=A_{0}$ and whether $B_{n}=D_{n}$. Since all the computational difficulties lie in $K_{2}$, we explain how we pursued the computations concerning $K_{2}$. A typical example will reveal the essential features of the computation. We take $K=\mathbb{Q}(\sqrt{-5219}), \mathfrak{p}=\mathbb{Z} 3+\mathbb{Z} \frac{1+\sqrt{-5219}}{2}$ and explain several techniques which were needed for our computation.

\subsection{Construction of $K_{2}$}

First we note that $h_{K}=24$ and $\mathfrak{p}$ is totally ramified in $K_{\infty}$. Set

$$
\tilde{f}_{j}(\mathfrak{c})=\left(g\left(\frac{r}{27}, \frac{s}{27}\right)\left(\frac{\omega_{1}}{\omega_{2}}\right) / g\left(\frac{4^{j} r}{27}, \frac{4^{j} s}{27}\right)\left(\frac{\omega_{1}}{\omega_{2}}\right)\right)^{4}
$$

with an ideal $\mathfrak{c}$ of $K$ and $1 \leq j \leq 8$, where $\mathfrak{p}^{3} \mathfrak{c}^{-1}=$ $\mathbb{Z} \omega_{1}+\mathbb{Z} \omega_{2}$ and $r \omega_{1}+s \omega_{2}=27$. Note that $\tilde{f}_{j}(\mathfrak{c})$ depends only on $\mathfrak{c}$. Let $C_{0}=\operatorname{cl}_{3}\left(\mathfrak{O}_{K}\right)$ and $\mathfrak{c}_{1}, \mathfrak{c}_{2}, \cdots, \mathfrak{c}_{24}$ be representatives of $I_{K} / P_{K}$ such that $\mathfrak{c}_{i}^{24}=\left(\gamma_{i}\right)$ with $\gamma_{i}^{2} \equiv 1$ $\left(\bmod \mathfrak{p}^{4}\right)$. Then we see that

$$
N_{K_{2}^{\prime} / K_{2}}\left(g_{\mathfrak{p}^{3}}\left(C_{0}\right)^{1-\sigma^{j}}\right)=\prod_{i=1}^{24} \tilde{f}_{j}\left(\mathfrak{c}_{i}\right)^{81},
$$

where $\sigma=\left(\frac{K_{2}^{\prime} / K}{4}\right)$. Set

$$
\beta_{j}=\zeta_{81} \prod_{i=1}^{24} \tilde{f}_{j}\left(\mathfrak{c}_{i}\right)
$$

with a $81^{\text {th }}$ root of unity $\zeta_{81}$. Lemma 3.2 implies that $\beta_{j}$ is contained in $K_{2}$ if we choose a suitable $\zeta_{81}$ for each $j$. We determine $\zeta_{81}$ so that the coefficients of

$$
\prod_{i=0}^{8}\left(X-\beta_{j}^{\sigma^{i}}\right)\left(X-\beta_{j}^{\sigma^{i} J}\right)
$$

which is the minimal polynomial of $\beta_{j}$ over $\mathbb{Q}$, are close to rational integers, where $J$ is the complex conjugation and the action of $\sigma$ for $\zeta_{81}$ is given by $\zeta_{81}^{\sigma}=\zeta_{81}^{16}$. As a result of these computations, we get $\zeta_{81}=1$ for each $j$.

Next we verify computationally that one of the $4^{\text {th }}$ roots of each $\beta_{j}$ is contained in $K_{2}$ (4.5). We put $\varepsilon=$ $\sqrt[4]{\beta_{4}}$. Then $\varepsilon$ is a unit of $K_{2}$ and the minimal polynomial $f(X)$ of $\varepsilon$ over $\mathbb{Q}$ has the least discriminant among $\sqrt[4]{\beta_{j}}$. Even though the coefficients of $f(X)$ are large, we show $f(X)$ completely for readers who are interested in this type of computation:

$$
\begin{aligned}
& f(X)=X^{18}-2737 X^{17}+169351307431 X^{16} \\
& +3928242055446129 X^{15}+1116673438382601450882 X^{14} \\
& -797848048872200987503002 X^{13} \\
& +14260371350698925012657372513 X^{12} \\
& +6727443351204545237345329632872 X^{11} \\
& +915274675664831410074802593822617 X^{10} \\
& +1633312619603207976653110097584811 X^{9} \\
& +1123545275437128223875406900453517 X^{8} \\
& -433121476304848342832840903771975 X^{7} \\
& +23565623970778493517049315349313 X^{6} \\
& +1799278132239867573207777918138 X^{5} \\
& +31191572789333418743352081696 X^{4} \\
& -9611439809099451726571366 X^{3} \\
& +1427400245427766872971 X^{2}+74348908961 X+1 .
\end{aligned}
$$

\subsection{Integral Basis of $\boldsymbol{K}_{\mathbf{2}}$}

Now we compute an integral basis of $K_{2}$ over $\mathbb{Z}$. We first try using KASH or PARI, however these packages cannot compute an integral basis due to the huge discriminant of $f(X)$. So we construct $\mathfrak{O}_{K_{2}}$ in the following way.

We start with $\mathbb{Z}[\varepsilon]$. By Lemma 2.5 , we see that

$$
\left(\mathfrak{O}_{K_{2}}: \mathbb{Z}[\varepsilon]\right)=\sqrt{\frac{|d(f)|}{\left|d\left(K_{2}\right)\right|}} \approx 2.1 \cdot 10^{394}
$$


Namely $\mathbb{Z}[\varepsilon]$ is a very small submodule of $\mathfrak{O}_{K_{2}}$. We can enlarge $\mathbb{Z}[\varepsilon]$ dramatically by adding a conjugate of $\varepsilon$. Set $M_{1}=\mathbb{Z}[\varepsilon]+\mathbb{Z} \varepsilon^{\sigma}$. Then $\left(\mathfrak{O}_{K_{2}}: M_{1}\right)=3^{6}$. Next we set

$$
M_{2}=\mathbb{Z}[\varepsilon]+\sum_{i, j} \mathbb{Z}{\sqrt[4]{\beta_{j}}}^{\sigma^{i}}
$$

Then we have $\left(\mathfrak{O}_{K_{2}}: M_{2}\right)=3$. Now we examine whether

$$
\varepsilon^{a_{0}+a_{1} \sigma+a_{2} \sigma^{2}+\cdots+a_{7} \sigma^{7}}
$$

is a cube in $K_{2}$ for integers $0 \leq a_{i} \leq 2$ using a method which will be explained in Section 4.5. We find that

$$
\varepsilon_{1}=\sqrt[9]{\varepsilon^{2+7 \sigma+6 \sigma^{2}+8 \sigma^{3}+4 \sigma^{4}+3 \sigma^{5}+5 \sigma^{6}+\sigma^{7}}}
$$

is contained in $K_{2}$. Finally we set $M_{3}=M_{2}+\mathbb{Z} \varepsilon_{1}$, yielding $\mathfrak{O}_{K_{2}}=M_{3}$.

\subsection{Unit Group of $K_{2}$}

The next task is a construction of the unit group $E_{K_{2}}$ of $K_{2}$. For all practical purposes, we only need a subgroup $E^{\prime}$ of $E_{K_{2}}$ with finite index prime to 3 .

We start with $E=\left\langle\varepsilon, \varepsilon^{\sigma}, \cdots, \varepsilon^{\sigma^{7}}\right\rangle$. In many cases, $E$ becomes a subgroup of $E_{K_{2}}$ with a finite index. If the index is infinite, we add $\sqrt[4]{\beta_{j}}$ to $E$ and obtain a subgroup of finite index. It is easy to enlarge $E$ to $E^{\prime}$ with an index prime to 3 , because $E_{K_{2}}$ has a small free rank 8 .

In the case $K=\mathbb{Q}(\sqrt{-5219})$, we see that $E^{\prime}=$ $\left\langle\varepsilon, \varepsilon^{\sigma}, \cdots, \varepsilon^{\sigma^{6}}, \varepsilon_{1}\right\rangle$ is a subgroup whose index is prime to 3 .

\section{$4.4 \mathrm{D}_{2}$ and $\mathrm{H}_{0,2}$}

As we have seen in the proof of Corollary 2.3, $H_{0, n}=A_{0}$ imples $B_{n}=D_{n}$. Hence, the calculation of $H_{0, n}$ is not needed to verify that $\lambda_{p}=0$. But we are interested in the least $n$ which satisfies the equalities $H_{0, n}=A_{0}$ or $B_{n}=D_{n}$.

We present a method which is applicable to the case $\left|A_{0}\right|=3$. It is easy to modify this for other cases. If $\left|D_{0}\right|=3$, then $\lambda_{3}=0$ from Proposition 2.2. So we assume $\left|D_{0}\right|=1$.

Let $\mathfrak{p}^{h^{\prime}}=(\alpha)$ with $h^{\prime}=h_{K} / 3$ and let $A_{0}=\langle\operatorname{cl}(\mathfrak{q})\rangle$ with $\mathfrak{q}^{3}=(\beta)$. Furthermore, let $E^{\prime}=\left\langle\varepsilon_{1}, \varepsilon_{2}, \cdots, \varepsilon_{8}\right\rangle$ be a subgroup of $E_{K_{2}}$ with index prime to 3 . Then we can determine $\left|D_{2}\right|$ and $\left|H_{0,2}\right|$ using the following lemmas.

Lemma 4.1. If

$$
\left(\alpha \prod_{i=1}^{8} \varepsilon_{i}^{e_{i}}\right)^{1 / 9}
$$

is contained in $K_{2}$ for some $0 \leq e_{i} \leq 8$, then $\left|D_{2}\right|=1$. Otherwise, $\left|D_{2}\right|=3$.

Lemma 4.2. If

$$
\left(\beta \prod_{i=1}^{8} \varepsilon_{i}^{e_{i}}\right)^{1 / 3}
$$

is contained in $K_{2}$ for some $0 \leq e_{i} \leq 2$, then $\left|H_{0,2}\right|=3$. Otherwise, $\left|H_{0,2}\right|=1$.

Remark 4.3. The number of trials for Lemma 4.2 is at most $3^{8}$. We note that the number of trials for Lemma 4.1 is not $9^{8}$. We can reduce it to $2 \cdot 3^{8}$ by expressing $e_{i}=e_{i, 0}+3 e_{i, 1} \quad\left(0 \leq e_{i, j} \leq 2\right)$.

For an integer $\alpha$ of $K_{2}$, we can get $\sqrt[3]{\alpha}$ explicitly if it is contained in $K_{2}$ by a method explained in the next paragraph. But this method requires a factorization of polynomials whose calculation needs a few seconds. Therefore, we will need several hours for the calculation given in Lemma 4.2. We use the next lemma to avoid wasteful trials.

Lemma 4.4. Let $\left\{\ell_{1}, \ell_{2}, \ldots, \ell_{r}\right\}$ be a finite set of prime numbers which split completely in $K_{2}$ and take rational integers $a_{j}$ and $a_{i j}$, such that $\beta \equiv a_{j}\left(\bmod l_{j}\right)$ and $\varepsilon_{i} \equiv$ $a_{i j}\left(\bmod l_{j}\right)$, where $l_{j}$ is a prime factor of $\ell_{j}$ in $K_{2}$. If

$$
a_{j} \prod_{i=1}^{8} a_{i j}^{e_{i}}+\ell_{j} \mathbb{Z}
$$

is not a cube in $\left(\mathbb{Z} / \ell_{j} \mathbb{Z}\right)^{\times}$for some $j$, then (4-2) is not contained in $K_{2}$.

We use a similar criterion for (4-1) and also for $E^{\prime}$.

\subsection{Cubic Root}

We explain how to calculate $\sqrt[3]{\alpha}$ for an integer $\alpha$ of $K_{2}$. We need a submodule of $\mathfrak{O}_{K_{2}}$ with small index (e.g., $M_{1}, M_{2}$ in (4.2)). Though a submodule of small index is enough for our purpose, we explain using $\mathfrak{O}_{K_{2}}$ for simplicity.

Let $\left\{v_{1}, v_{2}, \cdots, v_{18}\right\}$ be an integral basis of $K_{2}$. If $\sqrt[3]{\alpha} \in K_{2}$, then we can get the coefficients of $\sqrt[3]{\alpha}$ by solving approximately simultaneous equations:

$$
\sum_{i=1}^{18} x_{i} v_{i}^{\rho}=\sqrt[3]{\alpha^{\rho}} \quad\left(\rho \in \operatorname{Emb}\left(K_{2}, \mathbb{C}\right)\right) .
$$

If $(4-3)$ does not have integral solutions, then $\sqrt[3]{\alpha} \notin$ $K_{2}$. This is a well-known method; it works well in the 


\begin{tabular}{|c|r||c|c||c|c||c|}
\hline$m$ & $h_{K}$ & $\left|H_{0,1}\right|$ & $\left|D_{1}\right|$ & $\left|H_{0,2}\right|$ & $\left|D_{2}\right|$ & $\lambda_{3}$ \\
\hline-2081 & 60 & 1 & 3 & 3 & 3 & 0 \\
-2138 & 42 & 1 & 1 & 1 & 3 & 0 \\
-2183 & 42 & 1 & 1 & 1 & 1 & $?$ \\
-2186 & 42 & 1 & 3 & 3 & 3 & 0 \\
-3206 & 60 & 1 & 1 & 1 & 3 & 0 \\
-3614 & 60 & 1 & 3 & 3 & 3 & 0 \\
-4574 & 96 & 1 & 1 & 1 & 3 & 0 \\
-4637 & 78 & 1 & 1 & 1 & 1 & $?$ \\
-4835 & 30 & 1 & 3 & 3 & 3 & 0 \\
-5219 & 24 & 1 & 1 & 1 & 3 & 0 \\
-5579 & 30 & 3 & 3 & 3 & 3 & 0 \\
-5813 & 78 & 1 & 3 & 3 & 3 & 0 \\
-5897 & 48 & 1 & 1 & 1 & 3 & 0 \\
-6077 & 48 & 1 & 1 & 1 & 3 & 0 \\
-6269 & 114 & 1 & 3 & 3 & 3 & 0 \\
-6761 & 132 & 1 & 1 & 1 & 1 & $?$ \\
-6983 & 57 & 1 & 3 & 3 & 3 & 0 \\
-7862 & 78 & 1 & 3 & 3 & 3 & 0 \\
-7907 & 21 & 1 & 1 & 1 & 1 & $?$ \\
-8459 & 42 & 1 & 3 & 3 & 3 & 0 \\
-9113 & 96 & 3 & 3 & 3 & 3 & 0 \\
\hline
\end{tabular}

TABLE 1. $A_{0} \cong \mathbb{Z} / 3 \mathbb{Z}$.

totally real case. However, in our case, since $K_{2}$ is totally imaginary, we have to consider a difference by cubic root of unity for each $\sqrt[3]{\alpha^{\rho}}$. Namely, we need $3^{18}$ trials, which is computationally intensive even for a modern computer.

We use the following method. First, we construct the minimal polynomial $f(X)$ of $\alpha$ over $\mathbb{Q}$. The degree of $f(X)$ is often 18. Next we factorize $f\left(X^{3}\right)$. If it is irreducible over $\mathbb{Q}$, then $\sqrt[3]{\alpha} \notin K_{2}$. If $f\left(X^{3}\right)$ has a factor $g(X)$ of degree 18 , then $\sqrt[3]{\alpha} \in K_{2}$. Furthermore, we choose approximate values of $\sqrt[3]{\alpha^{\rho}}$ so that $g\left(\sqrt[3]{\alpha^{\rho}}\right)=0$ and get coefficients of $\sqrt[3]{\alpha}$ by solving (4-3).

\section{EXPERIMENTATION FOR $p=3$}

We show the result of the calculations which we have done in the case $p=3$. Let $K=\mathbb{Q}(\sqrt{m})$ with negative square free integer $m$. There exist $2282 \mathrm{~m}$ in the range $-10000<m<0$ such that (4-3) splits into $\mathfrak{p} \overline{\mathfrak{p}}$ in $K_{2}$. The distribution of $m$ is as follows:

\begin{tabular}{rcl}
\hline & number of $m$ & $\lambda_{3}$ \\
\hline$\left|A_{0}\right|=1$ & 1483 & 0 \\
$h_{k}=3$ & 4 & 0 \\
$h_{k}>3,\left|A_{0}\right|=3$ & 522 & $?$ \\
$\left|A_{0}\right|=9$ & 214 & $?$ \\
$\left|A_{0}\right|=27$ & 51 & $?$ \\
$\left|A_{0}\right|=81$ & 8 & $?$ \\
\hline
\end{tabular}

If $\left|A_{0}\right|=1$ or $h_{K}=3$, then $\lambda_{3}=0$. So we concentrate our attention on $522 m$ where $h_{K}>3$ and $\left|A_{0}\right|=3$. Let $A_{0}=\langle\operatorname{cl}(\mathfrak{q})\rangle$ with $\mathfrak{q}^{3}=(\beta)$. Then $\mathfrak{p}$ is totally ramified in $K_{\infty}$ if and only if $\beta^{2} \equiv 1\left(\bmod \mathfrak{p}^{2}\right)$. When $\mathfrak{p}$ is unramified in $K_{1} / K$, the genus formula implies $\left|A_{n}\right|=1$ for all $n \geq 1$ and consequently $\lambda_{3}=0$. Furthermore, when $\mathfrak{p}$ is totally ramified in $K_{\infty}$, then $\left|A_{0}\right|=\left|D_{0}\right|$ implies $\lambda_{3}=0$. The situation is summarized in the following table.

\begin{tabular}{ccl}
\hline $\mathfrak{p}$ & number of $m$ & $\lambda_{3}$ \\
\hline unramified in $K_{1}$ & 398 & 0 \\
totally ramified in $K_{\infty},\left|D_{0}\right|=3$ & 103 & 0 \\
totally ramified in $K_{\infty},\left|D_{0}\right|=1$ & 21 & $?$ \\
\hline
\end{tabular}

The number of targets for our experiments is 21 . We show the results of the calculations for $K_{1}$ and $K_{2}$ in Ta-

\begin{tabular}{|r|r||c||c|c||c|c||c|}
\hline$m$ & $h_{K}$ & $\left|D_{0}\right|$ & $\left|H_{0,1}\right|$ & $\left|D_{1}\right|$ & $\left|H_{0,2}\right|$ & $\left|D_{2}\right|$ & $\lambda_{3}$ \\
\hline-7265 & 72 & 3 & 1 & 9 & 3 & 9 & 0 \\
-17786 & 234 & 3 & 3 & 3 & 3 & 3 & $?$ \\
-19238 & 90 & 3 & 1 & 9 & 3 & 9 & 0 \\
-19466 & 234 & 3 & 1 & 9 & 3 & 9 & 0 \\
-19862 & 126 & 3 & 1 & 9 & 3 & 9 & 0 \\
-23231 & 234 & 3 & 1 & 9 & 3 & 9 & 0 \\
-23666 & 180 & 3 & 1 & 9 & 3 & 9 & 0 \\
-29402 & 144 & 3 & 3 & 3 & 3 & 9 & 0 \\
-34319 & 279 & 3 & 1 & 9 & 3 & 9 & 0 \\
-39335 & 198 & 1 & 3 & 3 & 3 & 9 & 0 \\
-41927 & 171 & 3 & 1 & 9 & 3 & 9 & 0 \\
-43415 & 144 & 3 & 1 & 9 & 3 & 9 & 0 \\
-45893 & 126 & 3 & 1 & 9 & 3 & 9 & 0 \\
-48266 & 198 & 1 & 1 & 3 & 1 & 9 & 0 \\
-48470 & 144 & 3 & 1 & 9 & 3 & 9 & 0 \\
-50846 & 360 & 3 & 1 & 9 & 3 & 9 & 0 \\
-54602 & 180 & 3 & 3 & 9 & 3 & 9 & 0 \\
-55067 & 90 & 3 & 1 & 9 & 3 & 9 & 0 \\
-65105 & 288 & 3 & 1 & 9 & 3 & 9 & 0 \\
-70223 & 315 & 1 & 3 & 3 & 9 & 9 & 0 \\
-76307 & 72 & 3 & 1 & 9 & 3 & 9 & 0 \\
-76469 & 396 & 3 & 3 & 3 & 9 & 9 & 0 \\
-78341 & 306 & 3 & 1 & 9 & 3 & 9 & 0 \\
-82442 & 342 & 1 & 1 & 3 & 1 & 9 & 0 \\
-83147 & 72 & 3 & 1 & 9 & 3 & 9 & 0 \\
-85019 & 144 & 3 & 1 & 9 & 3 & 9 & 0 \\
-88709 & 360 & 3 & 1 & 9 & 3 & 9 & 0 \\
-91895 & 288 & 1 & 1 & 3 & 1 & 9 & 0 \\
-92654 & 396 & 1 & 1 & 3 & 1 & 9 & 0 \\
-94631 & 414 & 3 & 1 & 9 & 3 & 9 & 0 \\
-97946 & 414 & 1 & 1 & 3 & 1 & 9 & 0 \\
-98009 & 252 & 1 & 1 & 3 & 1 & 9 & 0 \\
-99041 & 504 & 3 & 3 & 3 & 3 & 9 & 0 \\
\hline
\end{tabular}

TABLE 2. $A_{0} \cong \mathbb{Z} / 9 \mathbb{Z}$. 
ble 1, which seem to support a positive answer to Problem 1.1.

Our next trial is an experiment for $K$ with $\left|A_{0}\right|=9$. Since the treatment for $K$ with noncyclic $A_{0}$ is delicate, we restricted our targets to cyclic cases. There exist 197 $m$ such that $A_{0} \cong \mathbb{Z} / 9 \mathbb{Z}$ and $\mathfrak{p}$ is totally ramified in $K_{\infty}$ in the range $-100000<m<0$. We see $\lambda_{3}=0$ for $164 m$ verifying that $\left|D_{0}\right|=9$. Data for the $33 m$ with $\left|D_{0}\right| \leq 3$ is summarized in Table 2. This also suggests a positive answer to Problem 1.1.

Remark 5.1. Problem 1.1 is related to GGC (Generalized Greenberg Conjecture). Indeed, Minardi proved that if $\mathfrak{p}$ is totally ramified in $K_{\infty} / K$ and $\lambda_{p}=0$, then GGC holds for $K$ ([Minardi 86], [Ozaki 01]). So our examples are also examples for which GGC holds.

All the calculations in this paper were done by TC, which is available from $\mathrm{ftp}: / /$ tnt.math.metrou.ac.jp/pub/math-packs/tc/. The Alpha $21264667 \mathrm{MHz}$ needed 2 minutes for $m=-5219$, which is the easiest and 114 minutes for $m=-99041$, which is the hardest.

It is a natural question to ask the growth of the order of $A_{n}$ in the cases of Table 1 and 2. PARI succeeded in computing $A_{1}$ for small $m$. We report that $\left|A_{1}\right|=9$ for all $K$ in Table 1. It is difficult to compute $A_{2}$ or $\left|A_{2}\right|$ using PARI. Note that the proof of Lemma 2.2 implies $\left|A_{n}\right|=9 \quad(n \geq 1)$ for $K$ in Table 1 with $\left|D_{1}\right|=3$.

\section{REFERENCES}

[Gillard 85] R. Gillard. "Fonctions $L$ p-adiques des corps quadratiques imaginaires et de leurs extensions abéliennes." J. reine angew. Math. 358 (1985), 76-91.
[Greenberg 76] R. Greenberg. "On the Iwasawa Invariants of Totally Teal Number Fields." Amer. J. Math. 98 (1976), $263-284$

[Iwasawa 56] K. Iwasawa. "A Note on Class Numbers of Algebraic Number Fields." Abh. Math. Sem. Hamburg 20 (1956), 257-258.

[Kubert and Lang 81] D. S. Kubert and S. Lang. Modular Units, Grundlehren der mathematischen Wissenschaften Vol. 244. Berlin-Heidelberg: Springer Verlag, 1981.

[Minardi 86] J. Minardi. Iwasawa Modules for $\mathbb{Z}_{p}^{d}$-Extensions of Algebraic Number Fields. Thesis, University of Washington, 1986.

[Ozaki 01] M. Ozaki. "Iwasawa Invariants of $\mathbb{Z}_{p}$-Extensions over an Imaginary Quadratic Fields." In Class Field Theory - Its Centenary and Prospect, Advanced Studies in Pure Mathematics, Vol. 30, pp. 387-399, Singapore: World Scientific, 2001.

[Schertz 97] R. Schertz. "Construction of Ray Class Fields by Elliptic Units." Theorie des Nombres Bordeaux (1997), 383-394.

[Schneps 1987] L. Schneps. "On the $\mu$-invariant of $p$-adic $L$-functions Attached to Elliptic Curves with Complex Multiplication." J. Number Theory 25 (1987), 20-33.

[Stevenhagen 2001] P. Stevenhagen. Hilbert's 12th Problem, Complex Multiplication and Shimura Reciprocity,In Class Field Theory-Its Centenary and Prospect, Advanced Studies in Pure Mathematics, vol. 30, pp. 161176, Singapore: World Scientific, 2001. 387-399.

[Stark 1980] H. M. Stark. " $L$-functions at $s=1 . "$ Adv. Math. 35 (1980), 197-235.

[Yokoi 1967] H. Yokoi. "On the Class Number of a Relatively Cyclic Field." Nagoya Math. J. 29 (1967), 31-44.

Takashi Fukuda, Department of Mathematics, College of Industrial Technology, Nihon University, 2-11-1 Shin-ei, Narashino, Chiba, Japan (fukuda@math.cit.nihon-u.ac.jp)

Keiichi Komatsu, Department of Information and Computer Science, School of Science and Engineering, Waseda University, 3-4-1 Okubo, Shinjuku, Tokyo 169, Japan (kkomatsu@mse.waseda.ac.jp)

Received Febraury 7, 2002; accepted in revised form November 13, 2002. 